\title{
The residual gravity acceleration effect in the Poincaré dodecahedral space
}

\author{
B. F. Roukema and P. T. Różański \\ Toruń Centre for Astronomy, Nicolaus Copernicus University, ul. Gagarina 11, 87-100 Toruń, Poland
}

Received 19 February 2009 / Accepted 17 March 2009

\begin{abstract}
Context. In a flat space, it has been shown heuristically that the global topology of comoving space can affect the dynamics expected in the weak-field Newtonian limit, inducing a weak acceleration effect similar to dark energy.

Aims. Does a similar effect occur in the case of the Poincaré dodecahedral space, which is a candidate model of comoving space for solving the missing fluctuations problem observed in cosmic microwave background all-sky maps? Moreover, does the effect distinguish the Poincaré space from other well-proportioned spaces?

Methods. The acceleration effect in the Poincare space $S^{3} / I^{*}$ is studied, using a massive particle and a nearby test particle of negligible mass. Calculations are made in $S^{3}$ embedded in $\mathbb{R}^{4}$. The weak-limit gravitational attraction on a test particle at distance $r$ is set to be $\propto\left[R_{\mathrm{C}} \sin \left(r / R_{\mathrm{C}}\right)\right]^{-2}$ rather than $\propto r^{-2}$, where $R_{\mathrm{C}}$ is the curvature radius, in order to satisfy Stokes' theorem. A finite particle horizon large enough to include the adjacent topological images of the massive particle is assumed. The regular, flat, 3-torus $T^{3}$ is re-examined, and two other well-proportioned spaces, the octahedral space $S^{3} / T^{*}$, and the truncated cube space $S^{3} / O^{*}$, are also studied.

Results. The residual gravity effect occurs in all four cases. In a perfectly regular 3-torus of side length $L_{a}$, and in the octahedral and truncated cube spaces, the highest order term in the residual acceleration is the third-order term in the Taylor expansion in powers of $r / L_{a}$ (3-torus), or $r / R_{\mathrm{C}}$, respectively. However, the Poincaré dodecahedral space is unique among the four spaces. The third order cancels, leaving the fifth order term $\sim \pm 300\left(r / R_{\mathrm{C}}\right)^{5}$ as the most significant.

Conclusions. Not only are three of the four perfectly regular well-proportioned spaces better balanced than most other multiply connected spaces in terms of the residual gravity acceleration effect by a factor of about a million (setting $r / L_{a}=r / R_{\mathrm{C}} \sim 10^{-3}$ ), but the fourth of these spaces is about ten thousand times better balanced than the other three. This is the Poincaré dodecahedral space. Is this unique dynamical property of the Poincare space a clue towards a theory of cosmic topology?
\end{abstract}

Key words. cosmology: theory - cosmological parameters - large-scale structure of Universe - early Universe

\section{Introduction}

It has been shown that for zero curvature, the global topology of comoving space can affect the dynamics expected in the weakfield Newtonian limit (Roukema et al. 2007), in contrast to what was previously thought. In particular, a test particle of negligible mass near a massive particle (such as a cluster of galaxies dominated by its dark matter halo) has unequal attractions to the nearest topological images of the massive particle in opposite directions, since its position is asymmetrically offset from the massive particle. This leaves a residual acceleration effect that is qualititatively similar to that of dark energy. For realistic physical scales at the present epoch, the effect was estimated to be about $10^{-9}$ times weaker than the observed cosmological constant.

The heuristic calculations presented in Roukema et al. (2007) only considered the flat spaces $T \times \mathbb{R}^{2}$ and $T^{3}$. However, there has been interest in the Poincaré dodecahedral space, $S^{3} / I^{*}$, being a candidate model for comoving space that explains the "missing fluctuations problem" tentatively observed in the COsmic Microwave Background (COBE) all-sky maps and confirmed in the Wilkinson Microwave Anisotropy Probe (WMAP) all-sky maps (Spergel et al. 2003; Luminet et al. 2003; Roukema et al. 2004; Aurich et al. 2005a,b; Gundermann 2005; Key et al. 2007; Niarchou \& Jaffe 2007; Caillerie et al. 2007;
Lew \& Roukema 2008; Roukema et al. 2008a,b). Does a similar residual gravity effect occur in the case of positively curved space, in particular, in the Poincaré space?

An additional question of interest is whether or not the residual gravity effect might distinguish the Poincaré space from other well-proportioned spaces (Weeks et al. 2004). The Poincaré space is presently preferred to the octahedral space $\left(S^{3} / T^{*}\right)$ and the truncated cube space $\left(S^{3} / O^{*}\right)$ because of empirical constraints on curvature. For a total density of $1.02 \gtrsim \Omega_{\mathrm{tot}} \gtrsim 1.01$, the latter two spaces have larger fundamental domains than the Poincaré space and so have difficulty explaining the missing fluctuations problem. However, this is an empirical argument with no theoretical motivation. Could there be any dynamical arguments either favouring or disfavouring the Poincaré space? Some steps have been taken towards what might develop into a quantum cosmology theory of cosmic topology, using various notions of distance between different manifolds (Masafumi 1996; Anderson et al. 2004) and analysis of topology change in quantum gravity (e.g., Dowker \& Surya 1998). The residual gravity effect might contribute an additional criterion for comparing different manifolds.

Here, the residual gravity effect in the octahedral space, the truncated cube space, and the Poincaré space is studied by considering the dynamics of a negligible-mass test particle near a 
massive particle. As in Roukema et al. (2007), this approach can be considered as an heuristic model for a positively curved space that is homogeneous except for a small neighbourhood around one point, in which a positive density fluctuation of matter has collapsed into a high-density, nearly point-like object, in excess of the underlying homogeneous density distribution. To satisfy Stokes' theorem, the weak-limit gravitational attraction in the three spherical spaces is set to be proportional to

$$
\left[R_{\mathrm{C}} \sin \left(\frac{r}{R_{\mathrm{C}}}\right)\right]^{-2}
$$

rather than $r^{-2}$ (see Sect. 2.1), where $R_{\mathrm{C}}$ is the curvature radius. Numerical calculations are made in the universal covering space $S^{3}$ embedded in $\mathbb{R}^{4}$ for convenience. Accelerations are calculated in the tangent 3 -space at the position of the test particle, represented as 4-vectors in $\mathbb{R}^{4}$. The $T^{3}$ model discussed in Roukema et al. (2007) is revisited, using the covering space $\mathbb{R}^{3}$.

The method is described in more detail in Sect. 2. Residual accelerations estimated to the third order in the Taylor expansion of the fractional displacement are presented for the 3-torus in Sect. 3.1. Residual accelerations to fifth order for individual pairs of opposite images in the spherical spaces are presented analytically in Sect. 3.2. Residual accelerations to fifth order for the full set of adjacent ${ }^{1}$ images in the spherical spaces are presented numerically in Sect. 3.3. For the Poincaré space, an analytical derivation of the residual acceleration to fifth order is also given in Sect. 3.3.1. A statistical summary is presented in Sect. 3.3.2.

Discussion and conclusions are given in Sects. 4 and 5 respectively. Discussion of spherical, multiply connected spaces is available in Weeks (2001), Gausmann et al. (2001), Lehoucq et al. (2002), and Riazuelo et al. (2004), and we refer the reader to references therein for introductions to cosmic topology. Distances are calculated in a spatial section at constant cosmological time in the universal covering space $\left(S^{3}\right.$ for the spherical spaces, $\mathbb{R}^{3}$ for $T^{3}$ ), by default as "physical" distances, i.e., $r=$ $a \chi$, where $a(t)$ is the scale factor and $\chi$ is a comoving distance (i.e. $\chi$ is a "proper distance" at the present epoch (Weinberg 1972), equivalent to "conformal time" if $c=1$ ). Distance units are normally presented here in $h^{-1} \mathrm{Mpc}$ or $h^{-1} \mathrm{Gpc}$, where the Hubble constant is written $H_{0} \equiv 100 h \mathrm{~km} \mathrm{~s}^{-1} \mathrm{Mpc}^{1}$. The fundamental domains of all the spaces are assumed to be perfectly regular. The Newtonian gravitational constant is written as $G$.

\section{Method}

Although dynamics in the Poincaré dodecahedral space $S^{3} / I^{*}$ could, in principle, be studied by applying boundary conditions that identify opposite faces of a dodecahedral fundamental domain of a positive curvature radius $R_{\mathrm{C}}$ with one another, this would probably be extremely complicated for both analytical and numerical calculations. It is simpler to work in the universal covering space $S^{3}$ represented as a subspace of $\mathbb{R}^{4}$.

As described in Sect. 2.1 of Roukema et al. (2007), several assumptions are required for this heuristic approach. Here, the corresponding assumptions are as follows:

the flat Newtonian approximation of gravity is replaced by the equivalent in positively curved space, as described in Eq. (1) above;

(2), (3) the covering space is $S^{3}$, which is not flat;

\footnotetext{
1 "Adjacent" is used here to refer to images in copies of the fundamental domain that share a face with the "original" copy of the fundamental domain.
}

(4) a finite particle horizon $R_{\text {hor }}$ that is just large enough to include all adjacent topological images is assumed;

(5), (6) identical to those in Roukema et al. (2007): the metric is assumed to be that for a perfectly homogeneous model of the same curvature, except that the distant, multiple copies of the local massive particle are considered to provide a contribution to the local gravitational potential that may not fully cancel;

(7), (8) are not needed here since the one-body problem in $S^{3}$ (Sect. 3.1.1, Roukema et al.) is divergent and only briefly mentioned in Sect. 2.1.

\subsection{Weak-limit gravity and divergences}

As stated in Eq. (1), to satisfy Stokes' theorem, the weak-limit gravitational attraction towards a single massive particle is set to be proportional to $\left[R_{\mathrm{C}} \sin \left(r / R_{\mathrm{C}}\right)\right]^{-2}$ rather than $r^{-2}$. This can be understood as follows.

Let $S^{3}$ be represented by a spherical coordinate system centred on the massive particle, so that the Friedmann-LemaîtreRobertson-Walker (FLRW) line element is written

$\mathrm{d} s^{2}=-c^{2} \mathrm{~d} t^{2}+\mathrm{d} r^{2}+\left[R_{\mathrm{C}} \sin \left(r / R_{\mathrm{C}}\right)\right]^{2}\left(\mathrm{~d} \theta^{2}+\cos ^{2} \theta \mathrm{d} \phi^{2}\right)$,

where the scale factor $a(t)$ is subsumed into $r$ and $R_{\mathrm{C}}$, since it is not needed explicitly. We can write the weak-limit gravitational acceleration towards the massive particle as the vector field

$f(r) \hat{\boldsymbol{r}}$

by symmetry, where $\hat{\boldsymbol{r}}$ is the unit radial vector in the tangent space at a radial "physical" distance $r$ from the origin along a spatial geodesic, and

$\lim _{r \rightarrow 0^{+}} \frac{-f(r) r^{2}}{G m}=1$

in order to agree with the flat-space Newtonian limit towards the massive particle of mass $m$. Let $V$ be the interior of a 2-sphere of radius $r$ centred at the massive particle. Applying Stokes' theorem to $f(r) \hat{\boldsymbol{r}}$ on $V$, which is a sub-manifold of $S^{3}$ with boundary $\partial V$, gives

$$
\begin{aligned}
\int_{V} \operatorname{div}\left[f\left(r^{\prime}\right) \hat{\boldsymbol{r}}\right] \mathrm{d} V & =\int_{\partial V} f\left(r^{\prime}\right) \hat{\boldsymbol{r}} \cdot \mathrm{d} \boldsymbol{S} \\
& =\int_{\partial V} f\left(r^{\prime}\right)\left[R_{\mathrm{C}} \sin \left(r^{\prime} / R_{\mathrm{C}}\right)\right]^{2} \mathrm{~d} \theta \cos \theta \mathrm{d} \phi \\
& =f(r)\left[R_{\mathrm{C}} \sin \left(r / R_{\mathrm{C}}\right)\right]^{2} \int_{\partial V} \mathrm{~d} \theta \cos \theta \mathrm{d} \phi \\
& =4 \pi\left[R_{\mathrm{C}} \sin \left(r / R_{\mathrm{C}}\right)\right]^{2} f(r),
\end{aligned}
$$

using the FLRW metric. Since the only source of the vector field $f(r) \hat{\boldsymbol{r}}$ is the single massive particle, the left-hand side of this equation equals its limit for arbitrarily small (non-zero) volumes, i.e.

$$
\begin{aligned}
\int_{V} \operatorname{div}\left[f\left(r^{\prime}\right) \hat{\boldsymbol{r}}\right] \mathrm{d} V & =\lim _{r \rightarrow 0^{+}} \int_{V} \operatorname{div}\left[f\left(r^{\prime}\right) \hat{\boldsymbol{r}}\right] \mathrm{d} V \\
& =\lim _{r \rightarrow 0^{+}} 4 \pi\left[R_{\mathrm{C}} \sin \left(r / R_{\mathrm{C}}\right)\right]^{2} f(r) \\
& =-4 \pi G m,
\end{aligned}
$$

using Eqs. (5) and (4) respectively. Equating the right-hand sides of Eqs. (5) and (6) gives the weak limit acceleration induced by a single massive particle in a positively curved space of radius $R_{\mathrm{C}}$

$\ddot{\boldsymbol{r}}=f(r) \hat{\boldsymbol{r}}=-\frac{G m \hat{\boldsymbol{r}}}{\left[R_{\mathrm{C}} \sin \left(r / R_{\mathrm{C}}\right)\right]^{2}}$. 
For instantaneous transmission of gravitational signals in an infinitely old, static, simply connected space, this implies two divergence problems.

Firstly, a single massive point particle of mass $m$ in a perfectly uniform and otherwise empty $S^{3}$ yields an infinite repulsive force at the antipode of the massive particle, $\operatorname{since} \sin (\pi)=$ 0 . More generally, an infinite repulsive (attractive) force exists for gravitational signals that have travelled odd (even) values of $j$ times the half-circumference $\pi R_{\mathrm{C}}$ from the massive object, i.e. $j / 2$ times around the whole space, where $j \in \mathbb{Z}$.

Secondly, even if we ignore the model of a zero-size point particle as an idealised fiction, a problem remains for a negligible-mass test particle near a massive particle. The test particle also experiences accelerations from signals that have travelled $j$ times around $S^{3}$ in the two different directions along the great circle passing through the test particle and the massive particle, where $j \in \mathbb{Z}$. This second divergence problem is similar to the divergence problem in flat, simply connected, infinitely sized, infinitely old, Newtonian space with instantaneous transmission of the gravitational signal. The total acceleration towards the massive particle would be

$$
\begin{aligned}
\ddot{r}_{\mathrm{S}^{3}}= & \sum_{j=0}^{\infty} G m\left(R_{\mathrm{C}} \sin \frac{r+2 j \pi R_{\mathrm{C}}}{R_{\mathrm{C}}}\right)^{-2} \\
& -\sum_{j=-\infty}^{-1} G m\left(R_{\mathrm{C}} \sin \frac{r+2 j \pi R_{\mathrm{C}}}{R_{\mathrm{C}}}\right)^{-2} \\
= & G m\left(R_{\mathrm{C}} \sin \frac{r}{R_{\mathrm{C}}}\right)^{-2}\left(\sum_{j=0}^{\infty} 1-\sum_{j=-\infty}^{-1} 1\right),
\end{aligned}
$$

which is clearly divergent.

The former divergence, i.e., the $j \pi R_{\mathrm{C}}$ singularity related to the zero size of the massive point particle and the nature of positive curvature, is clearly unphysical if we are interested in a smoothing length on the scale of a galaxy cluster. The latter divergence, i.e. that in Eq. (8), is removed by assumption (4) above. Assumption (4) also removes the $j \pi R_{\mathrm{C}}$ divergence. A finite particle horizon $R_{\text {hor }}$ just large enough to include the adjacent topological images is necessary for a residual gravity effect to occur, but it does not need to be as large as $\pi R_{\mathrm{C}}$. The adjacent topological images in $S^{3} / T^{*}, S^{3} / O^{*}$, and $S^{3} / I^{*}$ are at $(\pi / 3) R_{\mathrm{C}}$, $(\pi / 4) R_{\mathrm{C}}$ and $(\pi / 3) R_{\mathrm{C}}$, and $(\pi / 5) R_{\mathrm{C}}$ respectively. Moreover, observationally realistic estimates of the total density parameter ${ }^{2}$ are consistent with the range that is empirically interesting for the Poincaré space, $1.02 \gtrsim \Omega_{\mathrm{tot}} \gtrsim 1.01$. The range $1.02 \gtrsim \Omega_{\mathrm{tot}} \gtrsim$ 1.01 corresponds to a curvature radius of $20 h^{-1} \mathrm{Gpc} \lesssim R_{\mathrm{C}} \lesssim$ $30 h^{-1} \mathrm{Gpc}$ respectively. Hence, for the three well-proportioned, spherical spaces, the appropriate horizon distances for the arrival of gravitational signals from the adjacent topological images are in the range $10 h^{-1} \mathrm{Gpc} \lesssim R_{\text {hor }} \lesssim 30 h^{-1} \mathrm{Gpc}$, i.e. up to a few times the distance to the surface of last scattering $r_{\mathrm{SLS}} \approx 10 \mathrm{~h}^{-1} \mathrm{Gpc}$. As noted in Roukema et al. (2007), a moderate amount of inflation in the early Universe could be one way of satisfying assumption (4).

\footnotetext{
${ }^{2}$ For example, $\Omega_{\mathrm{tot}}=1.010_{-0.009}^{+0.016}$, from WMAP 3-year and Hubble Space Telescope $H_{0}$ key project data, $\Omega_{\text {tot }}=1.015_{-0.016}^{+0.020}$, from WMAP 3-year data and Supernova Legacy Survey supernovae type Ia data (Spergel et al. 2007); $\Omega_{\text {tot }}=1.03_{-0.06}^{+0.04}$, from WMAP 3-year and Arcminute Cosmology Bolometer Array Receiver ACBAR data (Reichardt et al. 2009).
}

\subsection{Residual gravity}

The weak-limit residual acceleration on a test particle at $\boldsymbol{r}$ near the massive particle of mass $m$ at 0 can be written

$$
\ddot{\boldsymbol{r}}=\sum_{j=1, d_{j} \equiv a \chi\left[r, g_{j}(0)\right]}^{j=N} G m\left(R_{\mathrm{C}} \sin \frac{d_{j}}{R_{\mathrm{C}}}\right)^{-2} \hat{\boldsymbol{r}}_{j},
$$

where $g_{j}=1, \ldots, N$ are the $N=8,14$, or 12 holonomies that map an arbitrary object in $S^{3} / T^{*}, S^{3} / O^{*}$, or $S^{3} / I^{*}$ respectively to its adjacent topological images, $a$ is the scale factor, $\chi(\boldsymbol{a}, \boldsymbol{b})$ is the comoving distance between two positions $\boldsymbol{a}, \boldsymbol{b}$ in the covering space $S^{3}, \hat{\boldsymbol{r}}_{j}$ is the unit vector in the tangent space to $S^{3}$ at $\boldsymbol{r}$ pointing along the geodesic towards $g_{j}(0)$, and $\ddot{\boldsymbol{r}}$ is the acceleration vector in the same tangent space. The appendices in Gausmann et al. (2001) provide a convenient procedure for calculating this, where a 3-manifold $S^{3} / \Gamma$ and holonomy group $\Gamma$ are modelled as a tiling and isometry group of $\mathbb{R}^{4}$ using quaternions and 4-dimensional matrices. The tangent space at $\boldsymbol{r}$ is a 3 -plane, modelled using vectors in $\mathbb{R}^{4}$. The unit tangent vector towards the $j$ th topological image of the massive particle can be written

$\hat{\boldsymbol{r}}_{j}=\frac{g_{j}(0)-\left(\widehat{g_{j}(0)} \cdot \widehat{\boldsymbol{r}}\right) \boldsymbol{r}}{\left\|g_{j}(0)-\left(\widehat{g_{j}(0)} \cdot \widehat{\boldsymbol{r}}\right) \boldsymbol{r}\right\|}$

where $\widehat{x} \equiv \boldsymbol{x} /\|\boldsymbol{x}\|$. A useful identity is

$$
\left(\sin \frac{d_{j}}{R_{\mathrm{C}}}\right)^{-2}=\left(\sin \left\{\operatorname{acos}\left[\widehat{g_{j}(0)} \cdot \widehat{\boldsymbol{r}}\right]\right\}\right)^{-2}=\left\{1-\left[\widehat{g_{j}(0)} \cdot \widehat{\boldsymbol{r}}\right]^{2}\right\}^{-1} \text {. }
$$

The curvature is set to $R_{\mathrm{C}} \equiv 25 \mathrm{~h}^{-1} \mathrm{Gpc}$ below unless stated otherwise. The horizon $R_{\text {hor }}$ is set sufficiently large to include the full set of adjacent images of the massive particle, i.e., values of $R_{\text {hor }}=1.1(\pi / 3) R_{\mathrm{C}}, 1.1(\pi / 3) R_{\mathrm{C}}$, and $1.1(\pi / 5) R_{\mathrm{C}}$ are used in Eq. (9) for $S^{3} / T^{*}, S^{3} / O^{*}$, and $S^{3} / I^{*}$, respectively.

\subsection{Numerical precision}

For the full set of adjacent topological images in the spherical spaces, Eq. (9) is evaluated primarily by a numerical approach, using double precision floating-point operations where sufficient, and arbitrary precision floating-point operations where necessary. Typical scales of interest, i.e., for typical test particles in a void of large-scale structure, are a few tens of megaparsecs. This gives $10^{-4}<r / R_{\mathrm{C}}<10^{-2}$ as a useful domain for finding the highest terms in the Taylor expansion of the residual gravity for $r \ll R_{\mathrm{C}}$, where $R_{\mathrm{C}}=25 h^{-1} \mathrm{Gpc}$ as stated above. By setting $R_{\mathrm{C}}=G=m=1$ internally in numerical calculations, nearly equal and opposite accelerations from nearly opposed topological images are each of approximately unity order, the fourth and fifth order terms at the lower limit $r / R_{\mathrm{C}}=10^{-4}$ should therefore be above the numerical noise limit, if the calculating precision is well below $\sim 10^{-16}$ and $10^{-20}$, respectively. These would require precision in the significand ${ }^{3}$ well above $\log _{2} 10^{16} \approx 53$ and $\log _{2} 10^{20} \approx 66$ bits respectively. According to the IEEE 754-1985 standard, double-precision floating-point numbers have 53-bit precision in the significand (including one implicit bit). This would at best provide just one bit of information for a fourth order term and no information for a fifth order term for $r / R_{\mathrm{C}}=10^{-4}$. For this reason, an arbitrary precision library is used here to examine higher order terms.

\footnotetext{
3 The IEEE 754-2008 standard recommends the term "significand" rather than "mantissa".
} 
Analytical calculations are also made for the Poincaré space. We also revisit the $T^{3}$ calculation made in Sect. 3.2 of Roukema et al. (2007), in order to consider higher order terms. The first order term was found to cancel for a perfectly regular $T^{3}$ model.

\section{Results}

\section{1. $T^{3}$ revisited}

In Sect. 3.2 of Roukema et al. (2007), an analytical estimate of the acceleration from all six adjacent images in a $T^{3}$ model was shown to be zero to first order when the three fundamental lengths of the model are exactly equal. However, the lower scatterings of points in Figs. 7 and 8 of that paper do not represent numerical error. Recalculation of Eqs. (11) to (15) of Roukema et al. (2007) to higher order shows that for perfectly equal fundamental lengths $L_{a}=L_{e}=L_{u}$, higher order terms in Eq. (15) do not all cancel. The lowest order non-cancelling terms are the third order terms

$$
\begin{gathered}
\left(\ddot{\boldsymbol{r}}_{\text {topo }}\right)_{(i, j, k) \in\{( \pm 1,0,0),(0, \pm 1,0),(0,0, \pm 1)\}}=\frac{7 G m}{L_{a}^{2}}\left[2 \epsilon_{x}^{3}-3 \epsilon_{x}\left(\epsilon_{y}^{2}+\epsilon_{z}^{2}\right),\right. \\
\left.2 \epsilon_{y}^{3}-3 \epsilon_{y}\left(\epsilon_{x}^{2}+\epsilon_{z}^{2}\right), 2 \epsilon_{z}^{3}-3 \epsilon_{z}\left(\epsilon_{x}^{2}+\epsilon_{y}^{2}\right)\right]
\end{gathered}
$$

where the massive particle is at the origin $(0,0,0)$ in the covering space $\mathbb{R}^{3}$ and the test particle is slightly displaced at $\left(x=\epsilon_{x} L_{a}, y=\epsilon_{y} L_{a}, z=\epsilon_{z} L_{a}\right)$. For test particles distributed isotropically around the massive particle, numerical integration of these third order terms gives the distribution of accelerations. This distribution has a mean and standard deviation radial acceleration of

$\ddot{r} \approx(0.00 \pm 6.11) \frac{G m}{L_{a}^{2}}\left(\frac{r}{L_{a}}\right)^{3}$

and a skewness $\approx 0.58\left(G m / L_{a}^{2}\right)\left(r / L_{a}\right)^{3}$, where $r \equiv \sqrt{x^{2}+y^{2}+z^{2}}$. This expression is consistent with the lower scatterings of points in Figs. 7 and 8 of Roukema et al. (2007). Here, Fig. 1 shows a similar numerical calculation, using the radial component of the sum of $1 / r^{2}$ accelerations for the eight adjacent images, calculated directly from Eq. (10) of Roukema et al. (2007) without using the Taylor expansion. The acceleration is shown as

$a_{3}(r) \equiv \frac{\ddot{r}}{G m / L_{a}^{2}}\left(\frac{r}{L_{a}}\right)^{-3}$

for convenience.

The analytical and numerical calculations are clearly consistent in showing that in the case of perfectly equal fundamental side lengths of a $T^{3}$ model, there $i$ indeed a residual topological gravity effect. The residual acceleration is anisotropic, depending on the relation between the test particle's displacement from the massive particle and the orientation of the fundamental directions. The positive skewness implies that even though the mean radial acceleration is close to zero, the mode and median are negative, i.e., test particles placed in random directions relative to the massive particle are more likely to be subject to a radial deceleration rather than a radial acceleration. The amplitude of the effect is third order, i.e., about $\left(r / L_{a}\right)^{2}$ times smaller than the residual effect that occurs for slightly unequal fundamental lengths. If an upper estimate for $r / L_{a}$ at the present epoch is used, then this is a factor of about a million.

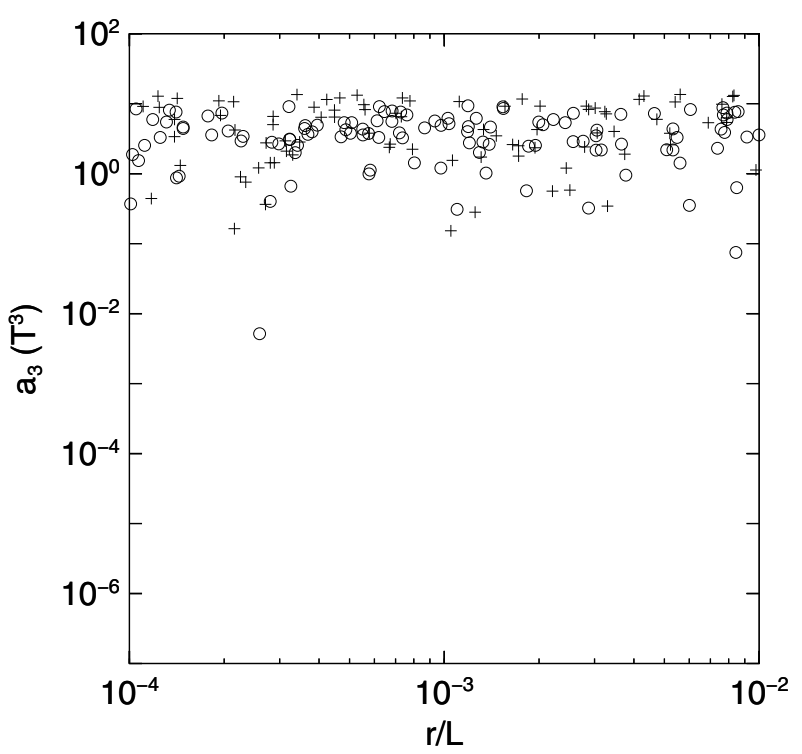

Fig. 1. Residual acceleration (radial component) induced on a test particle near a massive particle such as a cluster of galaxies by the six adjacent topological images of the massive particle, in a $T^{3}$ universe of three exactly comoving equal side lengths $L_{a}$. The accelerations $a_{3}(r)$ are normalised to be constant if the dominant term is third order in $r / L_{a}$ (see Eq. (14)) and are shown against distance $r<0.01 L_{a}$ from the massive particle, where $L=3000 h^{-1} \mathrm{Mpc}$. Positive accelerations are shown by plus symbols; negative accelerations are shown by circles.

\subsection{One pair of opposite topological images in $S^{3} / \Gamma$}

Before considering the full effect from a layer of topological images in the spherical cases, let us first consider the effect of just one pair of opposite topological images of the "local" massive particle. This is somewhat similar to the $T \times \mathbb{R}^{2}$ case considered in Sect. 3.1.1 of Roukema et al. (2007), where the test particle lies along the geodesic joining the three images of the massive particle to one another in one of the spherical spaces, i.e., $S^{3} / \Gamma$ for an appropriate holonomy group $\Gamma$. This may help us to understand the full sum from all the adjacent images. Similarly to Eq. (2) of Roukema et al. (2007), we can use scalar quantities. The acceleration to fifth order in $r / R_{\mathrm{C}}$ for the Poincaré space is ${ }^{4}$

$$
\begin{aligned}
\ddot{r}= & G \frac{m}{R_{\mathrm{C}}^{2}}\left\{\left[\sin \left(\frac{\pi}{5}-\frac{r}{R_{\mathrm{C}}}\right)\right]^{-2}-\left[\sin \left(\frac{\pi}{5}+\frac{r}{R_{\mathrm{C}}}\right)\right]^{-2}\right\} \\
= & G \frac{m}{R_{\mathrm{C}}^{2}}\left[\frac{4 \cos (\pi / 5)}{\sin ^{3}(\pi / 5)} \frac{r}{R_{\mathrm{C}}}\right. \\
& \left.+\frac{16 \cos (\pi / 5) \sin ^{2}(\pi / 5)+24 \cos ^{3}(\pi / 5)}{3 \sin ^{5}(\pi / 5)}\left(\frac{r}{R_{\mathrm{C}}}\right)^{3}+\ldots\right] \\
\approx & G \frac{m}{R_{\mathrm{C}}^{2}}\left[15.9\left(\frac{r}{R_{\mathrm{C}}}\right)+81.6\left(\frac{r}{R_{\mathrm{C}}}\right)^{3}+310.4\left(\frac{r}{R_{\mathrm{C}}}\right)^{5}+\ldots\right] .
\end{aligned}
$$

This is similar to the flat case. The residual acceleration is proportional to the displacement to first order, which again is behaviour similar to that of a cosmological constant:

$\ddot{r} \propto r$.

\footnotetext{
${ }_{4}$ The expression for the fifth order term is $\left[68 \cos (\pi / 5) \sin ^{4}(\pi / 5)+\right.$ $\left.240 \cos ^{3}(\pi / 5) \sin ^{2}(\pi / 5)+180 \cos ^{5}(\pi / 5)\right] /\left[15 \sin ^{7}(\pi / 5)\right]\left(\frac{r}{R_{C}}\right)^{5}$.
} 
The octahedral space has adjacent images at $(\pi / 3) R_{\mathrm{C}}$, so the corresponding calculation for a single pair of images is

$$
\begin{aligned}
\ddot{r} & =G \frac{m}{R_{\mathrm{C}}^{2}}\left\{\left[\sin \left(\frac{\pi}{3}-\frac{r}{R_{\mathrm{C}}}\right)\right]^{-2}-\left[\sin \left(\frac{\pi}{3}+\frac{r}{R_{\mathrm{C}}}\right)\right]^{-2}\right\} \\
& =\frac{16 \sqrt{3}}{9} G \frac{m}{R_{\mathrm{C}}^{2}}\left[\frac{r}{R_{\mathrm{C}}}+2\left(\frac{r}{R_{\mathrm{C}}}\right)^{3}+\frac{14}{5}\left(\frac{r}{R_{\mathrm{C}}}\right)^{5}+\ldots\right] \\
& \approx G \frac{m}{R_{\mathrm{C}}^{2}}\left[3.1\left(\frac{r}{R_{\mathrm{C}}}\right)+6.2\left(\frac{r}{R_{\mathrm{C}}}\right)^{3}+8.6\left(\frac{r}{R_{\mathrm{C}}}\right)^{5}+\ldots\right] .
\end{aligned}
$$

Equation (17) is also valid for the adjacent images at the eight truncated corners of the fundamental domain in a truncated cube space, which are at $(\pi / 3) R_{\mathrm{C}}$. The other six adjacent images in the truncated cube space are at $(\pi / 4) R_{\mathrm{C}}$, giving

$$
\begin{aligned}
\ddot{r} & =G \frac{m}{R_{\mathrm{C}}^{2}}\left\{\left[\sin \left(\frac{\pi}{4}-\frac{r}{R_{\mathrm{C}}}\right)\right]^{-2}-\left[\sin \left(\frac{\pi}{4}+\frac{r}{R_{\mathrm{C}}}\right)\right]^{-2}\right\} \\
& =8 G \frac{m}{R_{\mathrm{C}}^{2}}\left[\frac{r}{R_{\mathrm{C}}}+\frac{10}{3}\left(\frac{r}{R_{\mathrm{C}}}\right)^{3}+\frac{122}{15}\left(\frac{r}{R_{\mathrm{C}}}\right)^{5}+\ldots\right] \\
& \approx G \frac{m}{R_{\mathrm{C}}^{2}}\left[8\left(\frac{r}{R_{\mathrm{C}}}\right)+26.7\left(\frac{r}{R_{\mathrm{C}}}\right)^{3}+65.1\left(\frac{r}{R_{\mathrm{C}}}\right)^{5}+\ldots\right] .
\end{aligned}
$$

Each of these calculations is for just one pair of topological images adjacent to the "local" copy of the massive particle, lying in opposite directions. In the flat case, this can be thought of as a $T \times \mathbb{R}^{2}$ model, which is a valid 3-manifold. However, the spherical manifolds are not vector spaces, so a similar interpretation of the single-pair calculation is not possible.

On the other hand, Eqs. (15), (17), and (18) hint at the form that numerical estimates for the sum of the weak-limit residual gravitational effect from all the adjacent images may take for a given spherical manifold. For $S^{3} / I^{*}$, the full set of adjacent images of the massive particle consists of six pairs of images. For a test particle displaced slightly in a random direction from the massive particle, the two images in a pair will be seen in nearly, although not exactly, opposite directions in the tangent 3 -space at the test particle's location at $r \in S^{3}$. The modulus of the (vector) residual acceleration induced by the nearly opposite pair should not be very different from the expression given in Eq. (15), although its expression using elementary algebra might appear complicated.

This suggests that the scalar amplitude of the vector sum of all twelve accelerations is likely to involve terms of first, third, and fifth order in $r / R_{\mathrm{C}}$, with coefficients of the order of magnitude of those in Eq. (15). However, this argument is not exact. A test particle displaced from the massive particle in an arbitrary direction does not, in general, lie along the great circle defined by a given pair of opposite images, and can at most lie along only one of the great circles defined by the six pairs of opposite images. Hence, terms with even powers of $r / R_{\mathrm{C}}$ could also appear in the Taylor expansion.

These single-pair calculations might also be of interest for ill-proportioned (Weeks et al. 2004) positively curved spaces, e.g., the lens spaces $L(p, q)$, with $p, q \in \mathbb{Z}$ relatively prime, where $p \gg 1$ (e.g. Sect. 4, Gausmann et al. 2001). Since these spaces are not globally homogeneous, derivations similar to those in Eqs. (15), (17), and (18) would be strictly valid only for points lying along the symmetry axis joining the centres of the two faces of the fundamental dihedron (lens), i.e., where adjacent topological images are separated by a spatial geodesic of

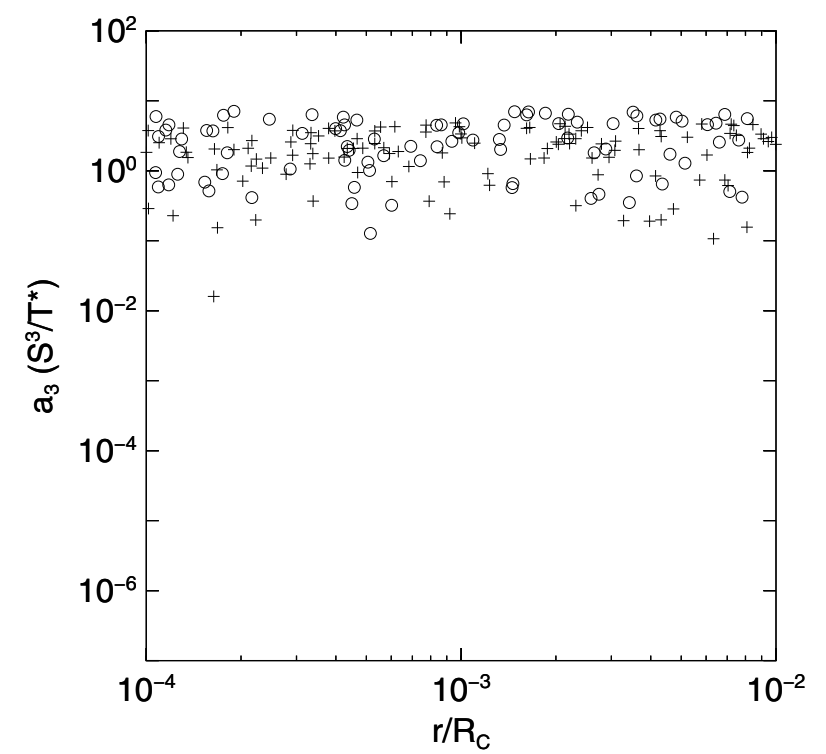

Fig. 2. Residual acceleration (radial component) induced on a test particle near a massive particle by the eight adjacent topological images of the massive particle, in a universe whose 3-manifold of comoving space is the octahedral space $S^{3} / T^{*}$. The accelerations $a_{3}(r)$ are normalised to be constant if the dominant term is third order in $r / L_{a}$ (see Eq. (19)) and are shown against distance $r<250 h^{-1} \mathrm{Mpc}=0.01 R_{\mathrm{C}}$ from the massive particle. Positive accelerations are shown by plus symbols; negative accelerations are shown by circles.

length $2 \pi R_{\mathrm{C}} / p$. This direction would therefore be unstable to a linear-term acceleration effect that would tend to expand it faster than other directions. This is qualitatively similar to the effect found in Sect. 3 of Roukema et al. (2007), according to which the residual acceleration would tend to equalise the three fundamental lengths of a $T^{3}$ model of slightly unequal fundamental lengths.

\subsection{Well-proportioned spherical spaces: $S^{3} / T^{*}, S^{3} / O^{*}$, and $S^{3} / l^{*}$}

As described in Sects. 2.2 and 2.3, for a test particle at $r$ in $S^{3} / T^{*}, S^{3} / O^{*}$, or $S^{3} / I^{*}$, let us set $R_{\mathrm{hor}}=1.1(\pi / 3) R_{\mathrm{C}}, R_{\mathrm{hor}}=$ $1.1(\pi / 3) R_{\mathrm{C}}$, or $R_{\mathrm{hor}}=1.1(\pi / 5) R_{\mathrm{C}}$, respectively. Equation (9) is evaluated numerically for 200 randomly (logarithmically) distributed test particles at distances of up to $250 h^{-1} \mathrm{Mpc}$ from the massive particle. Figures 2-4 show the residual accelerations, scaled by $\left(r / R_{\mathrm{C}}\right)^{3}$ so that they should be approximately constant if the dominating term in $r / R_{\mathrm{C}}$ is the third order term, i.e.,

$a_{3}(r) \equiv \frac{\ddot{r}}{G m / R_{\mathrm{C}}^{2}}\left(\frac{r}{R_{\mathrm{C}}}\right)^{-3}$

is shown.

It is clear that both the octahedral and truncated cube spaces have similar Taylor series behaviour to that of $T^{3}$. The linear term cancels, but a third-order-dominated residual acceleration remains. On the other hand, it is clear from Fig. 4 that the Poincare dodecahedral space has a residual acceleration that is much weaker than those of the other three spaces, and that this is poorly modelled as a third order term in $r / R_{\mathrm{C}}$. Instead, the constant slope of the relation in Fig. 4 strongly suggests that the 


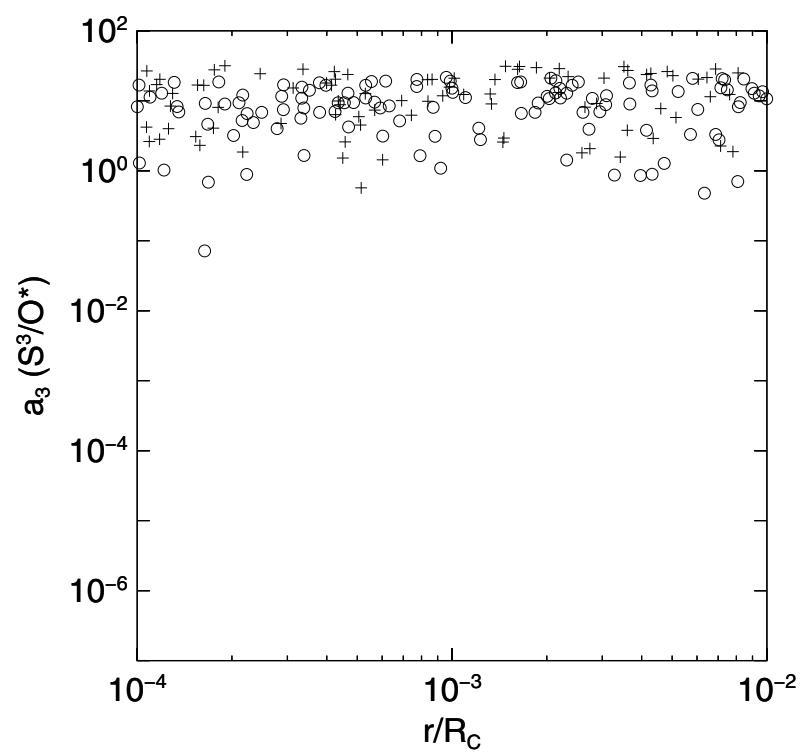

Fig. 3. As for Fig. 2, for the truncated cube space $S^{3} / O^{*}$.

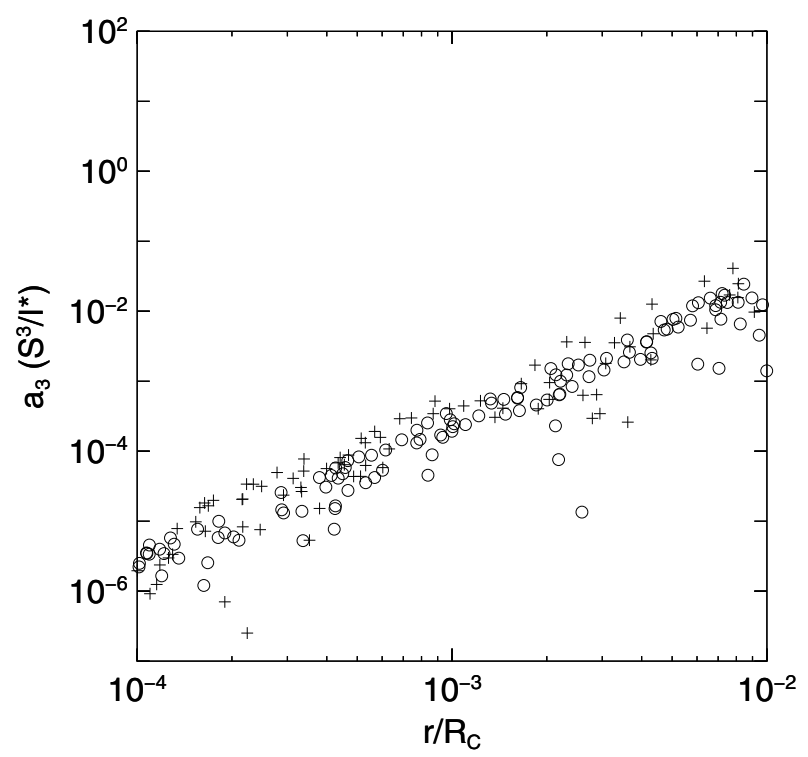

Fig. 4. Residual acceleration (radial component), as for Fig. 2, for the Poincaré dodecahedral space $S^{3} / I^{*}$.

residual acceleration for the Poincaré space is dominated by a fifth order term, i.e.,

$a_{5}(r) \equiv \frac{\ddot{r}}{G m / R_{\mathrm{C}}^{2}}\left(\frac{r}{R_{\mathrm{C}}}\right)^{-5}$

is approximately constant and terms lower than the fifth order cancel.

The remaining orthogonal component of the residual acceleration is of a similar order of magnitude to that of the radial component. Figure 5 shows that provided that the component is calculated with at least 70-bit precision in the significand, it is dominated by the $\left(r / R_{\mathrm{C}}\right)^{5}$ term.

The previous figures were calculated using 70-bit significand multi-precision floating-point operations. The effect of reducing the precision to 50 or 60 bits is clear in Fig. 5, i.e., for the orthogonal component of the residual acceleration in the Poincaré space. Noise created by the precision limit enters the calculation, for example, when converting from a double-precision position

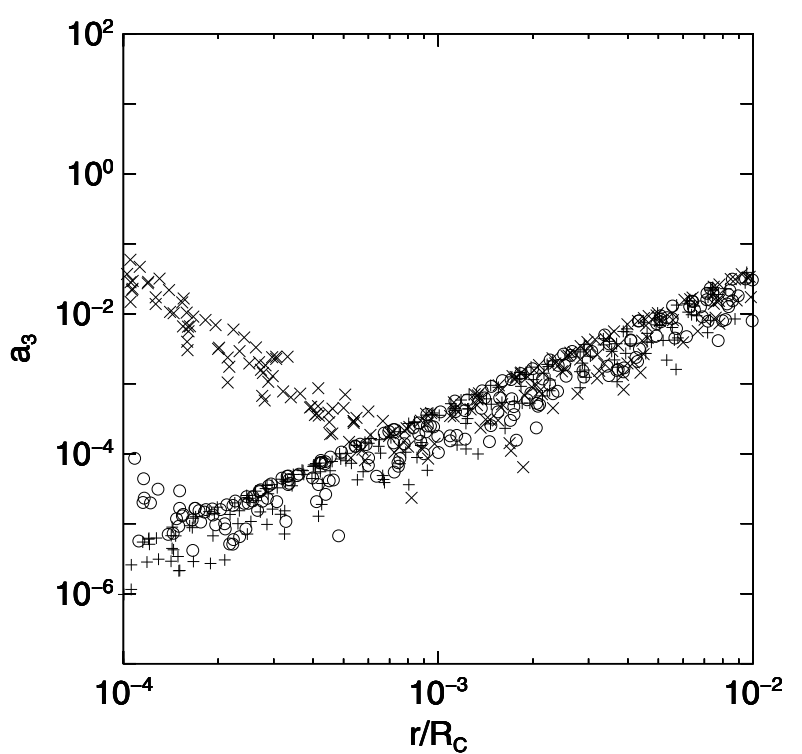

Fig. 5. Orthogonal residual acceleration for the Poincaré dodecahedral space $S^{3} / I^{*}$, as for Fig. 4, shown using 50-bit $(\times), 60$-bit (o) and 70-bit (+) precision in the significands of the floating-point numbers.

in $\mathbb{R}^{4}$ of the test particle to a multi-precision position. Apart from special cases, the finite precision representation of a typical, pseudo-random particle position in $\mathbb{R}^{4}$ will place the particle at a position slightly offset from the physical 3-surface $S^{3}$, because of the limited precision. If the particle is not located exactly on $S^{3}$, then this induces an acceleration that is orthogonal to the tangent 3-plane at $\boldsymbol{r}$. The numerical noise component appears on the left of Fig. 5 as a constant value of $\ddot{r}$, i.e., $a_{3}(r) \propto\left(r / R_{\mathrm{C}}\right)^{-3}$.

\subsubsection{Analytical estimate for the Poincaré space}

Analytical evaluation of Eq. (9) to fifth order for the Poincaré dodecahedral space using a computer algebra system ${ }^{5}$ confirms the numerical estimates shown in Figs. 4 and 5. The highest order residual acceleration from the adjacent topological images is

$$
\begin{aligned}
\ddot{\boldsymbol{r}}= & \frac{12 \sqrt{2}(297 \sqrt{5}+655)}{125 \sqrt{5-\sqrt{5}}}\left(\frac{r}{R_{\mathrm{C}}}\right)^{5} \\
& \left\{\left[70 y^{4}+(42 \sqrt{5}+70) x^{2} y^{2}-(14 \sqrt{5}+70) y^{2}\right.\right. \\
& \left.+(21 \sqrt{5}-7) x^{4}-28 \sqrt{5} x^{2}+7 \sqrt{5}+5\right] x, \\
& {\left[70 z^{4}+(42 \sqrt{5}+70) y^{2} z^{2}-(14 \sqrt{5}+70) z^{2}\right.} \\
& \left.+(21 \sqrt{5}-7) y^{4}-28 \sqrt{5} y^{2}+7 \sqrt{5}+5\right] y, \\
& {\left[70 x^{4}+(42 \sqrt{5}+70) x^{2} z^{2}-(14 \sqrt{5}+70) x^{2}\right.} \\
& \left.\left.+(21 \sqrt{5}-7) z^{4}-28 \sqrt{5} z^{2}+7 \sqrt{5}+5\right] z, 0\right\},
\end{aligned}
$$

where the massive particle is at $(0,0,0,1)$, the test particle displaced from it in an arbitrary direction by a small amount is located at $\left[\sin \left(r / R_{\mathrm{C}}\right) x, \sin \left(r / R_{\mathrm{C}}\right) y, \sin \left(r / R_{\mathrm{C}}\right) z, \cos \left(r / R_{\mathrm{C}}\right)\right]$, and $x^{2}+y^{2}+z^{2}=1$. The highest order term in the fourth component of this expression is a sixth order term in powers of $r / R_{\mathrm{C}}$.

\footnotetext{
5 The script is available online at http://adjani.astro.umk.pl/ GPL/dodec/PDS_residual. Version 1.0 was used in this paper.
} 
The fifth order exact expression can be written with approximate decimal coefficients as

$$
\begin{aligned}
\ddot{\boldsymbol{r}} \approx & \left(r / R_{\mathrm{C}}\right)^{5}\left[\left(4304.3 x^{4}+7540.5 y^{4}+17657.2 x^{2} y^{2}\right.\right. \\
& \left.-6744.5 x^{2}-10912.8 y^{2}+2224.7\right) x, \\
& \left(4304.3 y^{4}+7540.5 z^{4}+17657.2 y^{2} z^{2}\right. \\
& \left.-6744.5 y^{2}-10912.8 z^{2}+2224.7\right) y \\
& \left(4304.3 z^{4}+7540.5 x^{4}+17657.2 z^{2} x^{2}\right. \\
& \left.\left.-6744.5 z^{2}-10912.8 x^{2}+2224.7\right) z, 0\right] .
\end{aligned}
$$

The radial and orthogonal components of the residual acceleration can now be calculated as follows. Firstly, a small numerical value $r / R_{\mathrm{C}}=10^{-3}$ is chosen in order to evaluate the unit tangent vector at $\boldsymbol{r}$ pointing towards the massive particle. The sign of the latter is reversed in order to point away from the massive particle. Secondly, pseudo-random numerical values for $x, y, z$ are substituted into the full vector residual acceleration (Eq. (21) or (22)) and the scalar product with the unit tangent vector is taken in order to obtain the radial component of the residual acceleration. This is subtracted from the full residual to obtain the orthogonal component.

\subsubsection{Statistical description}

For an isotropic distribution of the directions of displacement of the test particle, Table 1 lists characteristic statistics of the radial and orthogonal components of the residual acceleration for the four well-proportioned spaces, primarily calculated from direct numerical estimates using Eq. (9). By construction, the orthogonal component is necessarily non-negative. Since estimation of the parameters of the Poincaré space residual acceleration is sensitive to numerical precision limits, Table 1 also lists parameters for the Poincaré space radial component estimated using Eq. (21) and the unit tangent vector described above. Within the uncertainties indicated by the standard error in the mean $\sigma_{\left\langle a_{i}\right\rangle}$, the two estimates agree.

\section{Discussion}

The results shown above are quite sensitive to small errors in the positions of the adjacent topological images. This provides a useful check of the calculations, since a small arbitrary error is most likely to yield a stronger residual acceleration than a weaker one. Increasing or decreasing the distances from the massive particle to the two members of one of the opposite pairs of topological images by $0.1 \%$ is sufficient to destabilise the nearly perfect equilibrium defined by the full set of adjacent topological images for a given space. The residual acceleration reverts to being dominated by $\left(r / R_{\mathrm{C}}\right)^{1}$ rather than $\left(r / R_{\mathrm{C}}\right)^{3}$ or $\left(r / R_{\mathrm{C}}\right)^{5}$.

This sensitivity to small changes in the distances to topological images is physically interesting. As found in Sect. 3 of Roukema et al. (2007), the residual acceleration in a $T^{3}$ model of slightly unequal fundamental lengths is dominated by a linear term in $r / L_{a}$, which tends to equalise the three fundamental lengths. The same relation applies for the spherical models. These are very well balanced in the sense that the linear term cancels, and in the case of the Poincare space, the cubic term cancels too, provided that these spaces are perfectly homogeneous. A small decrease in the injectivity diameter in one direction implies stronger acceleration for a test particle, favouring a return towards perfect isotropy in the fundamental lengths. Conversely, a small increase in the injectivity diameter implies weaker acceleration, again favouring a return towards isotropy.
Table 1. Statistical characteristics of coefficients $a_{i}$ of the dominant (ith order) term in the radial and orthogonal components of the residual acceleration $\ddot{r}$ in perfectly regular well-proportioned spaces, for approximately isotropic displacements $\boldsymbol{r}^{a}$.

\begin{tabular}{cccrrrr}
\hline \hline space & term $^{b}$ & $\| / \perp^{c}$ & $\left\langle a_{i}\right\rangle$ & $\sigma_{\left\langle a_{i}\right\rangle}^{d}$ & $\sigma_{i}^{e}$ & $\gamma_{i}^{f}$ \\
\hline 3-torus & $\left(r / L_{a}\right)^{3}$ & $\|$ & 0.00 & 0.00 & 6.11 & 0.58 \\
3-torus & $\left(r / L_{a}\right)^{3}$ & $\perp$ & 6.39 & 0.00 & 6.82 & -0.42 \\
octahedral & $\left(r / R_{\mathrm{C}}\right)^{3}$ & $\|$ & 0.01 & 0.01 & 3.18 & -0.58 \\
octahedral & $\left(r / R_{\mathrm{C}}\right)^{3}$ & $\perp$ & 3.33 & 0.00 & 1.26 & -0.42 \\
tr.cube & $\left(r / R_{\mathrm{C}}\right)^{3}$ & $\|$ & -0.05 & 0.05 & 14.24 & 0.58 \\
tr.cube & $\left(r / R_{\mathrm{C}}\right)^{3}$ & $\perp$ & 14.94 & 0.02 & 5.63 & -0.42 \\
dodec/num & $\left(r / R_{\mathrm{C}}\right)^{5}$ & $\|$ & -0.30 & 0.53 & 288.29 & 0.75 \\
dodec/alg & $\left(r / R_{\mathrm{C}}\right)^{5}$ & $\|$ & 0.00 & 0.01 & 288.26 & 0.74 \\
dodec/num & $\left(r / R_{\mathrm{C}}\right)^{5}$ & $\perp$ & 286.33 & 0.22 & 121.65 & -0.37 \\
\hline
\end{tabular}

${ }^{a}$ Coefficients $a_{i}$ as defined in Eqs. (14), (19), and (20); these are approximately constant with respect to $r$; the constant factor of $G m / L_{a}^{2}$ for the $T^{3}$ model or $G m / R_{\mathrm{C}}^{2}$ for the other models has been ignored here; all values shown are dimensionless.

${ }^{b}$ Dominant $i$ th power of displacement, as derived in this paper.

${ }^{c}$ Radial $\|$ or orthogonal $\perp$ component.

${ }^{d}$ Standard error in the mean $\sigma_{\left\langle a_{i}\right\rangle}=\sigma_{i} / \sqrt{N-1}$ for $N \gg 1$ test particles.

${ }^{e}$ Sample standard deviation.

${ }^{f}$ Sample skewness $\gamma_{i}=\left\langle\left[\left(a_{i}-\left\langle a_{i}\right\rangle\right) / \sigma_{i}\right]^{3}\right\rangle$.

${ }^{g}$ From 70-bit significand numerical calculations using Eq. (9).

${ }^{h}$ Using the algebraic expression in Eq. (21).

The residual acceleration tends to encourage the space to return to perfect "residual acceleration equilibrium", in which the residual acceleration disappears down to the third or fifth order.

Clearly, these effects are negligible in the present epoch at the level of observational detectability for the next few decades. Moreover, given the present empirical interest in the Poincaré space, the prediction of the perfectly isotropic model would be an absence of a residual acceleration effect to a much higher observational accuracy than that for the effects predicted by the other three well-proportioned models, which in turn would be more difficult to detect than the residual acceleration effect from ill-proportioned models.

However, the Universe is certainly inhomogeneous. If evidence continues to accumulate for the Poincaré space model with successively more accurate estimates of the model's astronomical coordinates, following the estimates made in Roukema et al. (2008a,b), then several observational approaches could be used to test the predictions of the residual acceleration effect. With a sufficient level of precision, matched circles and/or annuli on the sky should yield slightly different fundamental lengths along the six axes. The residual acceleration effect should consist of a positive or negative extra acceleration for shorter or longer fundamental lengths, respectively. Surveys of tracers of large-scale structure lying along the different geodesics to topological images of, for example, the Virgo cluster, could also be used to estimate which directions should have slightly longer than average geodesics and which should have slightly shorter than average geodesics. All three estimates should agree with one another, provided that no other sources of random and systematic error interfere. Hence, the residual acceleration effect potentially offers a physical mechanism for testing a multiplyconnected model of comoving space, as an alternative to relying on geometrical effects (individual or statistical identification of multiply imaged objects or regions of space). 
Another interesting question is whether or not the better "balancing" of the Poincaré space could have had any role in the evolution of a preferred topology in the early Universe, especially during the quantum epoch. Does the Poincaré space occupy a dynamically more stable state than the other wellproportioned spaces, which in turn are more stable than illproportioned spaces? This would most likely require significant inhomogeneities in the very early Universe, which themselves might have been subsequently removed by the residual gravity acceleration effect itself.

Apart from the possible physical consequences of this result, it may be interesting to ask if these results are reasonable from an intuitive mathematical point of view. It is useful to consider this in terms of pairs of opposite images, since if the test particle lies along the geodesic joining the local copy and the two opposite distant copies of the massive particle, then the single pair yields a linear-dominated residual acceleration. In order for the linear (or higher order) terms in the residual acceleration to cancel, the acceleration components that are approximately orthogonal to the geodesic joining the test particle to the two members of a given opposite pair need to be able to cancel the approximately radial components of residual accelerations in other pairs of images. In the $T^{3}$ case, three nearly orthogonal pairs are sufficient for the linear terms to completely cancel in this way (provided that the fundamental domain is perfectly regular). Given that $S^{3} / T^{*}, S^{3} / O^{*}$, and $S^{3} / I^{*}$ have four, seven, and six opposite pairs, respectively, it is reasonable to imagine that the radial components of the linear residual-acceleration term of a given pair are cancelled by the orthogonal components of the other pairs, since there are many of them, distributed symmetrically.

It is also possible to imagine that with a high enough number of opposite pairs and sufficient symmetry, a higher order term such as the third order might also disappear, by means of fine balancing between the different directions. The truncated cube space $S^{3} / O^{*}$ and the Poincare space $S^{3} / I^{*}$ have the highest numbers of opposite pairs, or equivalently, the highest numbers of pairs of faces of their fundamental domains. However, the truncated cube cannot be a regular polyhedron; its faces are six truncated squares and eight triangles. All the faces of the fundamental domain of the Poincaré dodecahedral space have the same shape: a pentagon. In this sense, it is more symmetrical than the truncated cube space. So requiring both a fundamental domain with a high number of faces and a high level of symmetry would seem to provide a qualitative pair of conditions to explain why the Poincaré space is more finely balanced than the other well-proportioned spaces.

However, could either the subset of topological images corresponding to only the octagonal faces of the fundamental domain of the truncated cube, or the subset corresponding to only the triangular faces, nevertheless cancel to fifth order, and become hidden because the other subset reintroduces a third order term? A calculation similar to those developed above shows that both subsets separately retain the third order as the dominant order. Table 2 gives statistical characteristics of the third order coefficients for the two subsets. So the Poincaré space retains its uniqueness in cancelling down to fifth order.

An aspect of the residual acceleration as expressed in Eqs. (21) and (22) that may initially seem counterintuitive is the permutational symmetry between the $x, y$, and $z$ components, since a dodecahedron is not always thought of as having cubical symmetry. However, an appropriate orientation of the dodecahedron in $\mathbb{R}^{3}$, such as that used in our computer algebra script, shows an $(x, y, z)$ symmetry. The twelve adjacent topological images consist of three quadruplets orthogonally
Table 2. Components of the residual acceleration $\ddot{r}$, as for Table 1, for the truncated cube space, separated by fundamental domain face shape.

\begin{tabular}{cccrrrr}
\hline \hline space & term & $\| / \perp$ & $\left\langle a_{i}\right\rangle$ & $\sigma_{\left\langle a_{i}\right\rangle}$ & $\sigma_{i}$ & $\gamma_{i}$ \\
\hline octagonal $^{a}$ & $\left(r / R_{\mathrm{C}}\right)^{3}$ & $\|$ & -0.00 & 0.04 & 17.46 & 0.58 \\
octagonal $^{b}$ & $\left(r / R_{\mathrm{C}}\right)^{3}$ & $\perp$ & 18.27 & 0.02 & 6.89 & -0.43 \\
triangular $^{b}$ & $\left(r / R_{\mathrm{C}}\right)^{3}$ & $\|$ & 0.00 & 0.01 & 3.19 & -0.58 \\
triangular & $\left(r / R_{\mathrm{C}}\right)^{3}$ & $\perp$ & 3.33 & 0.00 & 1.26 & -0.43 \\
\hline
\end{tabular}

${ }^{a}$ Contribution from the octagonal (truncated square) faces; ${ }^{b}$ contribution from the triangular faces.

projected to $\mathbb{R}^{3},[0, \pm 1 / 2, \pm(\sqrt{5}-1) / 4],[ \pm(\sqrt{5}-1) / 4,0, \pm 1 / 2]$, and $[ \pm 1 / 2, \pm(\sqrt{5}-1) / 4,0]$. Each of these quadruplets lies in a 2-plane in $\mathbb{R}^{3}$, i.e., $x=0, y=0$, and $z=0$, respectively. In this orientation, the "top" and "bottom" of the dodecahedron are edges, not faces. These two edges, along with four others, can be used to inscribe the dodecahedron in a cube (in flat space).

\section{Conclusions}

In Roukema et al. (2007), it was found that a residual gravity acceleration effect exists for $T \times \mathbb{R}^{2}$ but cancels to first order for a perfectly regular $T^{3}$ model. Here, it was found that the residual acceleration in $T^{3}$ does not completely cancel. In $T^{3}$ and two other well-proportioned spaces, the octahedral space $S^{3} / T^{*}$ and the truncated cube space $S^{3} / O^{*}$, the residual acceleration exists as a third order effect in the Taylor expansions in $r / L_{a}$ and $r / R_{\mathrm{C}}$ respectively. A reasonable upper limit to $r / L_{a}=r / R_{\mathrm{C}}$ can be set by considering test particles in voids of large-scale structure, i.e., at typically a few megaparsecs from the most massive nearby object, and an injectivity diameter for the 3manifold of comoving space of at least a few gigaparsecs, i.e., $r / L_{a}=r / R_{\mathrm{C}} \sim 10^{-3}$. Hence, at the present epoch, these three well-proportioned spaces are about a million times better balanced by this dynamical criterion than ill-proportioned spaces.

The Poincaré dodecahedral space $S^{3} / I^{*}$, presently a candidate 3-manifold for comoving space favoured by several observational analyses, has been found to be even more exceptional. Its residual acceleration is dominated by the fifth order term of amplitude $\sim \pm 300\left(G m / R_{\mathrm{C}}^{2}\right)\left(r / R_{\mathrm{C}}\right)^{5}$ (Figs. 4, 5, Table 1, Eqs. (21), (22)). This makes it about ten thousand times better balanced than the other three well-proportioned spaces, i.e., about $10^{10}$ times better balanced than ill-proportioned spaces. Moreover, perturbations to this equilibrium favour a return to the equilibrium state. Are these clues towards a theory of cosmic topology?

Acknowledgements. Thank you to Zbigniew Buliński and Bartosz Lew for helpful discussion and to an anonymous referee for useful suggestions. Use was made of the Centre de Données astronomiques de Strasbourg (http:// cdsads.u-strasbg.fr), the computer algebra program MAXIMA, the GNU OCTAVE command-line, high-level numerical computation software (http:// www.gnu.org/software/octave), the GNU multi-precision library (GMP) and the MPFR library, the GNU Scientific Library (GSL), and the GNU PLOTUTILS plotting package.

\section{References}

Anderson, M., Carlip, S., Ratcliffe, J. G., Surya, S., \& Tschantz, S. T. 2004, Class. Quant. Gra., 21, 729

Aurich, R., Lustig, S., \& Steiner, F. 2005a, Class. Quant. Gra., 22, 3443 Aurich, R., Lustig, S., \& Steiner, F. 2005b, Class. Quant. Gra., 22, 2061 
Caillerie, S., Lachièze-Rey, M., Luminet, J.-P., et al. 2007, A\&A, 476, 691

Dowker, F., \& Surya, S. 1998, Phys. Rev. D, 58, 124019

Gausmann, E., Lehoucq, R., Luminet, J.-P., Uzan, J.-P., \& Weeks, J. 2001, Class. Quant. Gra., 18, 5155

Gundermann, J. 2005 [arXiv: astro-ph/0503014]

Key, J. S., Cornish, N. J., Spergel, D. N., \& Starkman, G. D. 2007, Phys. Rev. D, 75,084034

Lehoucq, R., Weeks, J., Uzan, J.-P., Gausmann, E., \& Luminet, J.-P. 2002, Class. Quant. Gra., 19, 4683

Lew, B., \& Roukema, B. F. 2008, A\&A, 482, 747

Luminet, J., Weeks, J. R., Riazuelo, A., Lehoucq, R., \& Uzan, J. 2003, Nature, 425, 593

Masafumi, S. 1996, Phys. Rev. D, 53, 6902

Niarchou, A., \& Jaffe, A. 2007, Phys. Rev. Lett., 99, 081302

Reichardt, C. L., Ade, P. A. R., Bock, J. J., et al. 2009, ApJ, 694, 1200
Riazuelo, A., Weeks, J., Uzan, J., Lehoucq, R., \& Luminet, J. 2004, Phys. Rev. D, 69, 103518

Roukema, B. F., Lew, B., Cechowska, M., Marecki, A., \& Bajtlik, S. 2004, A\&A, 423,821

Roukema, B. F., Bajtlik, S., Biesiada, M., Szaniewska, A., \& Jurkiewicz, H. 2007, A\&A, 463, 861

Roukema, B. F., Buliński, Z., Szaniewska, A., \& Gaudin, N. E. 2008a, A\&A, 486, 55

Roukema, B. F., Buliński, Z., \& Gaudin, N. E. 2008b, A\&A, 492, 673

Spergel, D. N., Verde, L., Peiris, H. V., et al. 2003, ApJS, 148, 175

Spergel, D. N., Bean, R., Doré, O., et al. 2007, ApJS, 170, 377

Weeks, J. 2001, The Shape of Space, 2nd edn. (Manhattan: Marcel Dekker)

Weeks, J., Luminet, J.-P., Riazuelo, A., \& Lehoucq, R. 2004, MNRAS, 352, 258

Weinberg, S. 1972, Gravitation and cosmology: Principles and applications of the general theory of relativity (New York: Wiley) 